Revista de Psicología de la PUCP. Vol. XII. No 2. 1994

\title{
ALGUNAS ESTRATEGIAS UTILIZADAS POR FAMILIAS PERUANAS PARA AFRONTAR LA CRISIS ECONOMICA ACTUAL
}

Alegría Majluf ${ }^{1}$

Con el fin de explorar las estrategias urilizadas por madres de CSE media y baja para afrontar la crisis económica actual se entrevistaron a 20 madres de cada estrato económico y se les administraron tres pequeñas encuestas y la Escala F-Copes "Escala de Evaluación Personal del Funcionamiento Familiar en Situaciones de Crisis". Los resultados evidenciaron que ambos grupos económicos se vieron seriamente afectados económicamente debiendo reducir sus gastos en actividades vitales tales como alimentación, vestuario y recreación. Las madres de CSE media recurrieron más a una estrategia de reestructuración del problema para hacerlo más manejable y las madres de CSE baja a la movilización familiar para obtener ayuda y a la evaluación pasiva. No difieren ambos grupos en la búsqueda de apoyo espiritual y social.

Palabras claves: crisis socioeconómica; Escala F-Copes; CSE media y baja.

Some strategies used by Peruvian families to cope with the present socioeconomic crisis This study focuses on strategies used by mothers of middle and low SES to cope with the present socioeconomic crisis. Using a brief questionnaire and the F-Copes Scale, 20 mothers from each socioeconomic level were interviewed. Resules showed that both groups were affected economically and took measures to reduce their expenses in basic needs such as food, clothing and recreation. Mothers coming from the middle SES used estategies to reestructure the situation and make it more bearable, while mothers from low SES were more passive and relied on their extended families. No differences were found in their search for spiritual and social support.

Key words: socioeconomic crisis; F-Copes Scale; middle and low SES.

1. Profesora Principal en la Universidad Particular Cayetano Heredia y Profesora de la Pontificia Universidad Católica del Perú. Dirección José Quiñones 175, Lima 18. Realizó sus estudios en la Universidad Nacional Mayor de San Marcos y obtuvo el doctorado en psicología en la Universidad de Berkeley. Ha realizado importantes estudios y publicaciones en el campo de la psicología del desarrollo y marginalidad, publicando en 1993 un libro sobre "Marginalidad, Inteligencia y Rendimiento Escolar". Forma parte del comité editorial de la Revista de Psicología de la PUCP y es miembro del Colegio de Psicólogos y de varias sociedades profesionales extranjeras. 

Se ha comprobado que cambios excesivos abruman la capacidad física de reajuste y producen estrés. Eventos vitales tales como pérdida de un familiar, dificultades en el trabajo, crisis económica, se conciben como estresores que imponen cambios en los patrones básicos y habituales de vida.

El proceso activo de adaptación familiar, por ejemplo, a la crisis económica, implica estrategias para el manejo interno y para los patrones de interacción familia-comunidad, procesos que han recibido limitada atención en la investigación. Se advierte que esta carencia de estudios ocurre igualmente en el Perú, país que experimenta desde hace varias décadas múltiples problemas, siendo el más grave el de la crisis económica, la que se ha agudizado con el "shock" económico de 1990. Según encuesta de CUANTO (Marzo 1991) alrededor de 15 millones de personas, de una población total de 22 millones, se encuentran en situación de pobreza, 7 millones extrema pobreza y 5 millones en pobreza crítica. En Lima Metropolitana, el área más poblada y desarrollada del país, el $44 \%$ vive por debajo de la línea de pobreza.

Ante la dramática insuficiencia de recursos económicos que están afectando y dificultando el adecuado desarrollo no sólo de las familias empobrecidas, sino también de las familias "supuestamente" acomodadas se desconoce los medios o estrategias que éstas utilizan para afrontar la crisis.

Así mismo, se desconoce por qué unas familias son más vulnerables que otras o cómo varían en su capacidad de ajuste o solución de problemas. Por ello, el interés de explorar y conocer de qué estrategias se valen las familias peruanas de uno y otro estrato económico para afrontar la crisis. 
Ante esta problemática, inicialmente en Estados Unidos de Norteamérica, Hill (1958), desarrolló el modelo ABCX para comprender, en especial, por qué las familias enfrentadas con un evento estresor varían en su capacidad de ajuste. Dentro de este modelo, $A$ se refiere al evento estresor, el que interactúa con $B$, o sea recursos de la familia ante la crisis, el que a su vez interactúa con $C$, definición que hace la familia del evento, generando todo este proceso $X$, es decir, la crisis.

Trabajando con ese modelo $\mathrm{ABCX}$, expertos en familia han examinado la conducta familiar ante la crisis, entre ellos McCubbin, Larsen y Olson (1981) integran los factores de acumulación de fuentes de estrés, recursos familiares y sentido/percepción logrando desarrollar la Escala F - COPES, "Escala de Evaluación Personal del Funcionamiento Familiar en Situaciones de Crisis". Este tenía por objeto determinar la forma cómo la familia maneja sus problemas y cómo afronta las demandas que surgen en su medio, pero que afectan a la familia como unidad. Al término del desarrollo de la prueba (que será descrita en Metodología) los autores definieron las siguientes cinco Escalas: (1) Obtención de apoyo social por parte de parientes, amigos y vecinos; (2) Reestructuración o cómo la familia redefine los eventos estresantes para hacerlos más manejables; (3) Búsqueda de apoyo espiritual; (4) Movilización familiar para obtener y aceptar ayuda y; (5) Evaluación pasiva o aceptación del problema minimizando sus efectos.

Respecto al estudio a realizarse en Lima, de acuerdo a resultados de múltiples investigaciones, se asumió que familias de CSE media enfrentadas a una crisis tenderán a usar más estrategias enfocadas: (1) a la reestructuración del problema con el fin de hacerlo más manejable; (2) a la obtención de apoyo o búsqueda de información de profesionales, parientes y amigos y; (3) probablemente, también debido principalmente a la significación e importancia que tiene la religión en el país, recurran a la búsqueda de apoyo espiritual. Las familias pertenecientes a la CSE baja tratarán de obtener (1) apoyo de pariente, amigos y vecinos; (2) movilizarán a la familia para obtener y aceptar apoyo de programas comunitarios; (3) recurrirán, así mismo, por la razón señalada como las familias acomodadas, al apoyo espiritual y por último (4) quizás muchas de las familias harán una evaluación pasiva del problema minimizando sus efectos. 


\section{Metodología}

\section{Muestra}

Se aplicaron las encuestas a 20 madres de la CSE media y 20 madres de la CSE baja (Zonas Marginales), siendo la edad promedio en el primer grupo de: 38 años la madre y 41 ańos el padre; en el segundo grupo: 42 años la madre y 44 años el padre. La CSE media tiene como promedio 2 hijos, mientras la CSE baja tiene 3 hijos. El nivel educacional y ocupación de ambos grupos se consignan en los Cuadros 1 y 2.

Cuadro 1: Porcentaje del nivel educacional de los padres de CSE media y la CSE baja $(\mathrm{G} 1=20$ y G2 = 20)

\begin{tabular}{|lcccc|}
\hline & \multicolumn{2}{c}{ CSE media } & \multicolumn{2}{c|}{ CSE baja } \\
nivel educacional & padre & madre & padre & madre \\
& $\%$ & $\%$ & $\%$ & $\%$ \\
\hline superior & 85 & 70 & - & - \\
técnica & - & - & - & 5 \\
secundaria completa & 15 & 30 & 47 & 42 \\
secundaria incompleta & - & - & 24 & 11 \\
primaria & - & - & 29 & 42 \\
\hline
\end{tabular}

Cuadro 2: Porcentaje de la ocupación de los padres de CSE media $(\mathbf{n}=20)$ y CSE baja $(n=20)$

\begin{tabular}{|lcccc|}
\hline & \multicolumn{2}{c}{ CSE media } & \multicolumn{2}{c|}{ CSE baja } \\
ocupación & $\begin{array}{c}\text { padre } \\
\%\end{array}$ & $\begin{array}{c}\text { madre } \\
\%\end{array}$ & $\begin{array}{c}\text { padre } \\
\%\end{array}$ & $\begin{array}{c}\text { madre } \\
\%\end{array}$ \\
\hline profesional & 60 & 15 & - & - \\
empleado & 25 & 35 & - & - \\
comerciante & 5 & - & 18 & 11 \\
técnico & 5 & 5 & 12 & - \\
obrero & - & - & 64 & 21 \\
chofer & 5 & - & 6 & - \\
doméstica & - & - & - & 42 \\
su casa & - & 55 & - & 26 \\
\hline
\end{tabular}




\section{Instrumentos}

Se administraron a las 40 madres en forma individual una encuesta con tres preguntas para estimar: (1) sus ingresos en los últimos 6 meses; (2) su situación económica actual y; (3) las estrategias que utilizaron con más frecuencia para afrontar la crisis (los porcentajes obtenidos en cada una se presentan en Resultados en los Cuadros 3,4, y 5) y en Escala F COPES "Escala de Evaluación Personal del Funcionamiento Familiar en Situaciones de Crisis" que se describe a continuación.

La Escala F - COPES fue desarrollada por McCubbin, Larsen y Olson en 1981 para identificar la solución de problemas y las estrategias conductuales utilizadas por las familias en dificultades o situaciones de crisis. Los autores diseñaron la escala para integrar los recursos familiares y los factores de sentido/percepción establecidos en la teoría del estrés familiar (Hill, 1958; Burr, 1973; McCubbin y Patterson, 1982) y las estrategias de manejo de crisis.

Inicialmente generaron 49 ítemes, los que fueron probados con familias representativas de todos los estadios del ciclo vital y en base al análisis de calidad y varianza redujeron los ítemes a 29. Posteriormente realizaron un análisis factorial para determinar las dimensiones sobresalientes de las cuales surgieron ocho escalas. Con otras dos muestras de sujetos se aplicó otro análisis factorial con rotación varimax. La estructura factorial fue muy similar al análisis factorial inicial.

En base a los análisis realizados, los autores colapsaron en un solo factor los tres originales relativos a la obtención de apoyo social de la familia extensa, amigos y vecinos, la que denominaron: Obtención de Apoyo Social (factor 1). Ello, también ocurrió con dos factores originales, Confianza en la solución de problemas y Reestructuración que identificaron como Reestructuración (factor 2); Búsqueda de Apoyo Espiritual y Evaluación Pasiva se conservaron idénticos a los factores originales constituyendo los factores 3 y 4 . Por último, se integró el 5to. factor Movilización Familiar para Obtener apoyo y los recursos de la comunidad y Aceptación de regalos por parte de los vecinos. Los cinco factores tuvieron valores mayores que 1 y cada uno de los 29 ítemes tuvo un peso factorial mayor que 38. El Coeficiente Alfa de Cronbach se calculó para cada factor y para la Escala Total, teniendo esta última un coeficiente de .77 . 
La confiabilidad test-retest, con un lapso de tiempo entre la primera y segunda aplicación de 4 a 5 semanas, fue de .71. (La Encuesta, la Escala F - COPES y los ítemes que corresponden a cada factor de la Escala se presentan en el Anexo).

\section{Calificación}

Se obtiene el puntaje para cada una de las 5 escalas sumando los números marcados como respuesta (conforme a lo indicado en el protocolo de la prueba) a cada ítem por los encuestados. El puntaje total lo constituye la suma de los puntajes parciales logrados en las 5 escalas.

\section{Resultados}

Los porcentajes consignados en las Tablas 3, 4 y 5 evidencian, primero, que en relación a los ingresos en los últimos 6 meses (Cuadro 3) las madres de la CSE media señalan, por un lado, que se ha incrementado algo, aunque en la misma proporción manifiestan que ha disminuido mucho, y un $\mathbf{3 0}$ por ciento no han observado mayor cambio. En la CSE baja $21 \%$ de las madres reportan que sus ingresos se han incrementado algo, aunque también en la misma proporción dicen que ha disminuido mucho, observándose que este último porcentaje de madres quienes notan que sus ingresos han disminuido algo (36.8\%), constituyendo en conjunto un $58 \%$ de madres que indican que tienen problemas serios en su situación económica actual.

Cuadro 3: ¿Cuál de las siguientes afirmaciones describe mejor lo que ha ocurrido con sus ingresos los últimos 6 meses?

\begin{tabular}{|lcc|}
\hline & $\begin{array}{c}\text { CSE media } \\
\%\end{array}$ & $\begin{array}{c}\text { CSE baja } \\
\%\end{array}$ \\
\hline Se han incrementado mucho & - & 10.5 \\
Se han incrementado algo & 35 & 21 \\
Continúa lo mismo & 25 & 10.5 \\
Han disminuido algo & 5 & 36.8 \\
Han disminuido mucho & 35 & 21.1 \\
\hline
\end{tabular}


En cuanto a la situación económica actual (Cuadro 4) se advierte que concuerda con lo anteriormente señalado, ya que las madres de la CSE media en un $35 \%$ han experimentado problemas serios a muy serios y las de CSE baja reportan en un $58 \%$ problemas económicos muy serios.

Cuadro 4: ¿Cuál de las siguientes afirmaciones describe mejor la situación económica actual de su familia?

\begin{tabular}{|lcc|}
\hline & CSE media & CSE baja \\
$\%$ & $\%$ \\
\hline Ningún problema & 10 & 10.5 \\
Problemas leves & 55 & 31.5 \\
Problemas serios & 30 & 58 \\
Problemas muy serios & 5 & - \\
\hline
\end{tabular}

Para afrontar la crisis el Cuadro 5 evidencia que las madres de CSE media en un $100 \%$ (entre algunas veces y frecuentemente) han tenido que reducir sus gastos en recreación, vestuario, alimentación y además han tenido que tomar trabajos adicionales para ayudarse económicamente, notándose que las familias de la CSE baja, en la misma proporción, han tenido que reducir sus gastos en las áreas mencionadas, aunque en un porcentaje mucho más bajo han tomado trabajos adicionales, probablemente debido a la escasez de oportunidades de trabajo para este grupo que cuenta con limitada educación.

Cuadro 5: Durante los últimos 6 meses con qué frecuencia su familia

\begin{tabular}{|lccrr|}
\hline & $\begin{array}{c}\text { CSE } \\
\mathrm{A}^{\mathbf{2}} \\
\%\end{array}$ & $\begin{array}{c}\text { media } \\
\mathrm{B}^{\mathrm{b}} \\
\%\end{array}$ & $\begin{array}{r}\text { CSE } \\
\mathrm{A}^{\mathrm{a}} \\
\%\end{array}$ & $\begin{array}{r}\text { baja } \\
\mathrm{B}^{\mathrm{b}} \\
\%\end{array}$ \\
\hline Redujo gastos en recreación & 55 & 45 & 63.2 & 36.8 \\
Redujo compras de vestuario & 70 & 30 & 62.6 & 37.4 \\
Cambió hábitos de comida & 85 & 15 & 54.4 & 45.6 \\
Tomó trabajos adicionales & 50 & 50 & 26 & 74 \\
\hline
\end{tabular}

\footnotetext{
algunas veces

b Frecuentemente
} 
Respecto a los resultados en la Escala F - COPES (Cuadro 6) se observan diferencias significativas entre ambos grupos en las estrategias utilizadas, notándose que las familias de CSE media recurren más a la "reestructuración"; es decir, una redefinición de los eventos estresantes para hacerlos más manejables, mientras que las familias de CSE baja tienden a usar más la movilización familiar para obtener y aceptar ayuda, por ejemplo, de los comedores populares, cunas, etc., y a la "evaluación pasiva o aceptación del problema minimizando sus efectos". No se advierten diferencias entre ambos estratos en la obtención de apoyo social de parientes, amigos y vecinos, ni en la búsqueda de apoyo espiritual, concordando estos resultados con lo postulado en este estudio.

Cuadro 6: Promedios (X), desviaciones estándar (DS) y pruebas t para las diferencias entre las estrategias utilizadas para afrontar la crisis económica (F-COPES) por familias de clase media y clase baja

\begin{tabular}{|lrrrrr|}
\hline & CSE & media & CSE & baja \\
estrategias & $X$ & $D S$ & $X$ & \multicolumn{1}{c|}{$D S$} & $t$ \\
\hline reestructuración & 30 & 4 & 28 & 2 & $1.96^{*}$ \\
búsqueda de apoyo social & 23 & 6 & 26 & 4.3 & 1.76 \\
búsqueda de apoyo espiritual & 14.5 & 3 & 14 & 2 & 1.29 \\
movilización familiar para & & & & & \\
obtener y aceptar ayuda & 9.7 & 2.4 & 13.4 & 2 & $5.31^{* *}$ \\
evaluación Pasiva & 7.7 & 3 & 11 & 4.2 & $2.46^{*}$ \\
\hline
\end{tabular}

* $\mathrm{p}<.05$

** $p<.01$

\section{Discusión}

Los resultados, de carácter exploratorio, obtenidos en el presente estudio evidencian que la crisis económica actual ha afectado igualmente a las familias acomodadas como a las más empobrecidas, aunque en mayor grado a estas últimas; es decir, que ambos grupos han visto sus ingresos económicos reducidos severamente y acusan serios problemas. Ambos estratos redujeron sus gastos en actividades vitales esenciales, tales como alimentación, vestuario, recreación, aunque las familias más acomodadas han podido defenderse en alguna forma tomando trabajos adicionales, lo 
cual no pudieron lograrlo las familias más empobrecidas, probablemente debido a la escasez de oportunidades de trabajo.

Respecto a las otras estrategias que han utilizado las familias para afrontar la crisis se advierten diferencias significativas en la modalidad, recurriendo las de CSE media más a la reestructuración y las de CSE baja a la movilización familiar para obtener y aceptar ayuda a la evaluación pasiva, no difiriendo ambos estratos en la búsqueda de apoyo espiritual y de apoyo social.

Considerando el número reducido de familias estudiadas se sugeriría realizar otra investigación con una muestra más amplia y representativa para conocer en forma más detallada como ha afectado la crisis económica a las familias y las estrategias que han utilizado para afrontarla tanto en la Costa, como en la Sierra y en la Selva.

\section{A N E X O}

\section{ENCUESTA}

Durante estos años de crisis socio-económica por las que atraviesa el país todos los peruanos hemos sufrido, en mayor o menor grado, las consecuencias de esta crisis debiendo desarrollar una serie de alternativas y estrategias para afrontarlas. Nos interesaría, para fines de estudio de nuestra realidad, conocer las diversas formas que han empleado las personas para reducir el efecto de la crisis.

Por ejemplo:

I. ¿Cuál de las siguientes afirmaciones describe mejor lo que ha ocurrido con sus ingresos los últimos 6 meses?.
A. Se han incrementado mucho
B. Se han incrementado algo
C. Continúa lo mismo 

D. Han disminuído algo
E. Han disminuído mucho.

II. ¿Cuál de las siguientes afirmaciones describe mejor la situación económica actual de su familia?.
A. Ningún problema
B. Problemas leves
C. Problemas serios
D. Problemas muy serios

III. Durante los últimos 6 meses con qué frecuencia su familia (Responda marcando alguna de las 2 alternativas siguientes):
$A=$ algunas veces
$B=$ frecuentemente

1. Redujo gastos en recreación y actividades sociales. A B

2. Redujo compras de ropa y vestuario A B

3. Cambió hábitos de comida A B

4. Tomó trabajos adicionales para ayudarse en los gastos

A B

\section{MADRE}

Edad :

Educación :

Ocupación :

Nro. de hijos :

Edades :

\section{PADRE}

Edad:

Educación :

Ocupación : 


\section{F - COPES}

\section{ESCALA DE EVALUACION PERSONAL DEL FUNCIONAMIEN- TO FAMILIAR EN SITUACIONES DE CRISIS}

Hamilton I. McCubbin, David H. Olson, Andrea S. Larsen

\section{Instrucciones:}

Primero, lea la lista de "Alternativas de Respuestas", una a una. Luego decida qué tan bien cada frase describe sus actitudes y conductas en respuesta a problemas y dificultades. Si la frase describe muy bien su respuesta, tache el número 5, indicando que está en "MARCADO ACUERDO"; si la frase no describe para nada su respuesta, tache el número 1 , indicando que usted está en "MARCADO DESACUERDO"; si la frase describe su respuesta en alguna medida, seleccione el número 2,3 ó 4, para indicar qué tanto acuerdo o desacuerdo hay entre la frase y su respuesta.

\begin{tabular}{|c|c|c|c|c|c|}
\hline & \multicolumn{5}{|c|}{$\begin{array}{l}\text { ALTERNATIVAS } \\
\text { DE RESPUESTAS }\end{array}$} \\
\hline $\begin{array}{l}\text { CUANDO EN NUESTRA FAMILIA } \\
\text { ENFRENTAMOS PROBLEMAS O } \\
\text { DIFICULTADES, NOSOTROS } \\
\text { RESPONDEMOS: }\end{array}$ & 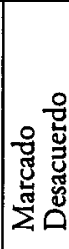 & 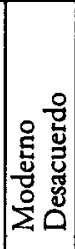 & 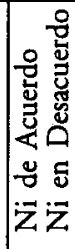 & 苞通 & 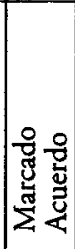 \\
\hline 1. Compartiendo nuestras dificultades con los parientes & 1 & 2 & 3 & 4 & 5 \\
\hline 2. Buscando estímulo y apoyo en los amigos & 1 & 2 & 3 & 4 & 5 \\
\hline $\begin{array}{l}\text { 3. Sabiendo que nosotros tenemos el poder para resol- } \\
\text { ver problemas importantes }\end{array}$ & 1 & 2 & 3 & 4 & 5 \\
\hline $\begin{array}{l}\text { 4. Buscando información y consejo de personas que } \\
\text { han enfrentado problemas iguales o parecidos }\end{array}$ & 1 & 2 & 3 & 4 & 5 \\
\hline 5. Buscando consejo en los parientes (abuelos, tíos etc.) & 1 & 2 & 3 & 4 & 5 \\
\hline $\begin{array}{l}\text { 6. Buscando asistencia en servicios y programas comu- } \\
\text { nitarios, diseñados para ayudar a familias en nuestra } \\
\text { tra situación }\end{array}$ & 1 & 2 & 3 & 4 & 5 \\
\hline $\begin{array}{l}\text { 7. Sabiendo que dentro de nuestra propia familia tene- } \\
\text { mos la fortaleza para resolver nuestros propósitos pro } \\
\text { problemas }\end{array}$ & 1 & 2 & 3 & 4 & 5 \\
\hline
\end{tabular}




\begin{tabular}{|c|c|c|c|c|c|c|}
\hline & $\begin{array}{l}\text { Recibiendo regalos y favores de los vecinos } \\
\text { (comida, que hagan diligencias, etc.) }\end{array}$ & 1 & 2 & 3 & 4 & 5 \\
\hline 9. & $\begin{array}{l}\text { Buscando información y consejo del médico de } \\
\text { la familia }\end{array}$ & 1 & 2 & 3 & 4 & 5 \\
\hline 10 & Pidiendo a los vecinos ayuda y favores & 1 & 2 & 3 & 4 & 5 \\
\hline 11. & $\begin{array}{l}\text { Enfrentando los problemas "con la cabeza" y tra- } \\
\text { tando de encontrar soluciones inmediatamente }\end{array}$ & 1 & 2 & 3 & 4 & 5 \\
\hline 12. & Viendo televisión & 1 & 2 & 3 & 4 & 5 \\
\hline 13. & Demostrando que somos fuertes & 1 & 2 & 3 & 4 & 5 \\
\hline 14. & Asistiendo a los servicios de la iglesia & 1 & 2 & 3 & 4 & 5 \\
\hline 15. & $\begin{array}{l}\text { Aceptando los eventos estresantes como parte de } \\
\text { la vida }\end{array}$ & 1 & 2 & 3 & 4 & 5 \\
\hline & $\begin{array}{l}\text { Compartiendo las preocupaciones con amigos } \\
\text { cercanos }\end{array}$ & 1 & 2 & 3 & 4 & 5 \\
\hline 17. & $\begin{array}{l}\text { Viendo en los juegos de suerte un recurso impor- } \\
\text { tante para resolver nuestros problemas familiares }\end{array}$ & 1 & 2 & 3 & 4 & 5 \\
\hline & $\begin{array}{l}\text { Aceptando que las dificultades aparecen en forma } \\
\text { inesperada }\end{array}$ & 1 & 2 & 3 & 4 & 5 \\
\hline & $\begin{array}{l}\text { Compartiendo actividades con parientes (salir } \\
\text { juntos, comer, etc.) }\end{array}$ & 1 & 2 & 3 & 4 & 5 \\
\hline 20. & $\begin{array}{l}\text { Buscando consejo profesional y ayuda para las } \\
\text { dificultades familiares }\end{array}$ & 1 & 2 & 3 & 4 & 5 \\
\hline & $\begin{array}{l}\text { Creyendo que nosotros podemos manejar nuestros } \\
\text { propios problemas }\end{array}$ & 1 & 2 & 3 & 4 & 5 \\
\hline 22. & Participando en las actividades de la iglesia & 1 & 2 & 3 & 4 & 5 \\
\hline 23. & $\begin{array}{l}\text { Definiendo el problema familiar en una forma } \\
\text { más positiva, de modo que no nos sintamos } \\
\text { demasiado desalentados }\end{array}$ & 1 & 2 & 3 & 4 & 5 \\
\hline 24. & $\begin{array}{l}\text { Preguntándole a los parientes cómo se sienten } \\
\text { ellos con los problemas que nosotros enfrentamos }\end{array}$ & 1 & 2 & 3 & 4 & 5 \\
\hline 25. & $\begin{array}{l}\text { Sintiendo que, sin importar lo que hagamos para } \\
\text { estar preparados, siempre tendremos dificultad } \\
\text { para afrontar los problemas }\end{array}$ & 1 & 2 & 3 & 4 & 5 \\
\hline 26. & Buscando consejo de un sacerdote (o religioso/a) & 1 & 2 & 3 & 4 & 5 \\
\hline & $\begin{array}{l}\text { Creyendo que si esperamos lo suficiente, el pro- } \\
\text { blema desaparecerá por sí solo }\end{array}$ & 1 & 2 & 3 & 4 & 5 \\
\hline 28. & Compartiendo los problemas con los vecinos & 1 & 2 & 3 & 4 & 5 \\
\hline 29. & Teniendo fe en Dios & 1 & 2 & 3 & 4 & 5 \\
\hline
\end{tabular}




\title{
ESCALA DEL F - COPES
}

\author{
Itemes que constituyen \\ cada Escala
}

\begin{abstract}
OBTENCION DEL APOYO SOCIAL. Nueve ítemes miden la habilidad de la familia para ocuparse activamente en la obtención de apoyo por parte de parientes, amigos, vecinos y familia extensa.
\end{abstract}

$1,2,4,5,10,16,20,25,29$

REESTRUCTURACION. Esta dimensión con ocho ítemes evalúa la capacidad de la familia para redefinir los eventos estresantes para hacerlos más manejables.

$3,7,11,13,15,19,22,24$

BUSQUEDA DE APOYO ESPIRITUAL. Cuatro ítemes enfocados sobre la habilidad para obtener apoyo espiritual.

$14,23,27,30$

MOVILIZACION FAMILIAR PARA OBTENERY ACEPTAR AYUDA. La habilidad familiar para buscar recursos en la comunidad y aceptar ayuda de otros, es medida por estos cuatro ítemes.

$6,8,9,21$ 


\section{Referencias}

Burr, W. (1973). Theory construction and the sociology of the family. Nueva York: John Wiley \& Sons.

Cuanto S.A. (1990). Perú en números. Lima: CUANTO S.A.

Hill, R. (1949). Families under stress. Nueva York: Harper \& Row.

Hill, R. (1958). Generic Features of families under stress. Social Casework, 213 - 258.

McCubbin, H. (1979). Integrating coping behavior in family under stress. Journal of Marriage and the Family, 41, 237 - 244.

McCubbin, H., Larsen, A. \& Olson, D. (1981). F-COPES. Escala de Evaluación Personal del Funcionamiento Familiar en Situaciones de Crisis. En D. Olson, H. McCubbin, H. Barnes, A. Larsen, M. Muxen, \& M. Wilson. (Eds.), Inventarios sobre Familia. Traducido por Angela Fernández (1989). Facultad de Psicología. Bogotá: Universidad Santo Tomás.

McCubbin, H. \& Patterson, J. (1982). Family adaptation to crisis. Illinois: Charles Thomas Publishers. 